\title{
Flight and attitude dynamics measurements of an instrumented Frisbee
}

\author{
Ralph D Lorenz \\ Lunar and Planetary Laboratory, University of Arizona, 1629 E University Blvd, Tucson, \\ AZ 85721, USA \\ E-mail: rlorenz@lpl.arizona.edu
}

Received 12 July 2004, in final form 7 December 2004

Published 11 February 2005

Online at stacks.iop.org/MST/16/738

\begin{abstract}
In-flight measurements are made of the translational accelerations and attitude motion of a hand-thrown flying disc using miniaturized accelerometers and other sensors and a microcontroller data acquisition system. The experiments explore the capabilities and limitations of sensors on a rapidly rotating platform moving in air, and illustrate several of the complex gyrodynamic aspects of Frisbee flight. The data give insight into the biomechanics of Frisbee launch, and indicate lift, drag and pitch moment coefficients consistent with previous wind-tunnel measurements. The experiments constitute an instructive exercise in aerospace vehicle systems integration and in attitude reconstruction, and open the way to guided disc wings using control surfaces actuated during specific spin phases determined by onboard sensors.
\end{abstract}

Keywords: attitude, orientation, dynamics, free-flight, accelerometer, magnetometer, sun sensor, video, aerodynamics, gyrodynamics, disc wing, Frisbee, spacecraft

\section{Introduction and motivation}

A remarkable feature of the flight of spin-stabilized discwings (most familiar in a recreational form such as the 'Frisbee', a trademark of Wham-O, Inc.) is that a wide range of nonmonotonic flight behaviours may be generated by manipulating the launch conditions (launch speed and elevation, angle of attack and spin rate). These are a result of the combined gyrodynamic and aerodynamic properties of the disc (e.g. $[1,2])$.

A disc may veer in one direction and then another, making a snake-like path. These behaviours are due to the variation of the disc's aerodynamic coefficients with both angle of attack (which varies throughout the flight as the disc's forward motion slows and falls downwards under gravity) and the advance ratio. This latter quantity - the ratio of the tip rotation speed to the forward speed of the vehicle-increases throughout the flight. As the rotation rate remains approximately constant while the forward speed slows, the advance ratio increases.

The aerodynamic properties of flying discs have only recently been comprehensively documented in wind-tunnel tests [3]. This work (see also [4]) has shown how the pitch moment coefficient, as well as the roll and sideforce coefficients, varies as the advance ratio increases to around unity. Note that the lift and drag coefficients are relatively insensitive to spin.

The work of Potts and Crowther [3], performed with the disc mounted on a motorized sting in a wind tunnel, spanned angles of attack between -10 and $30^{\circ}$ and advance ratios of up to 1.04. (Another report [4] by the same workers gives coefficients for zero spin up to $50^{\circ}$, including the stall point at $\sim 45^{\circ}$.) However, in recreational applications, a Frisbee may routinely fly at angles of attack up to $90^{\circ}$ in a conventional throw, and indeed up to $-90^{\circ}$ in throws such as the 'hammer' where the disc is thrown at a slightly negative angle of attack, to roll onto its back and descend near vertically in an inverted attitude. Furthermore, in some real flight conditions when the forward speed becomes very low, the advance ratio may increase substantially above unity.

In order to explore these flight regimes and to investigate the capabilities of on-board measurements, we have undertaken experiments with free-flying discs equipped with on-board sensors and data acquisition equipment.

An additional motivation for these tests is the evaluation of attitude and trajectory determination methods for planetary probes. These, such as the Mars Pathfinder probe or the 


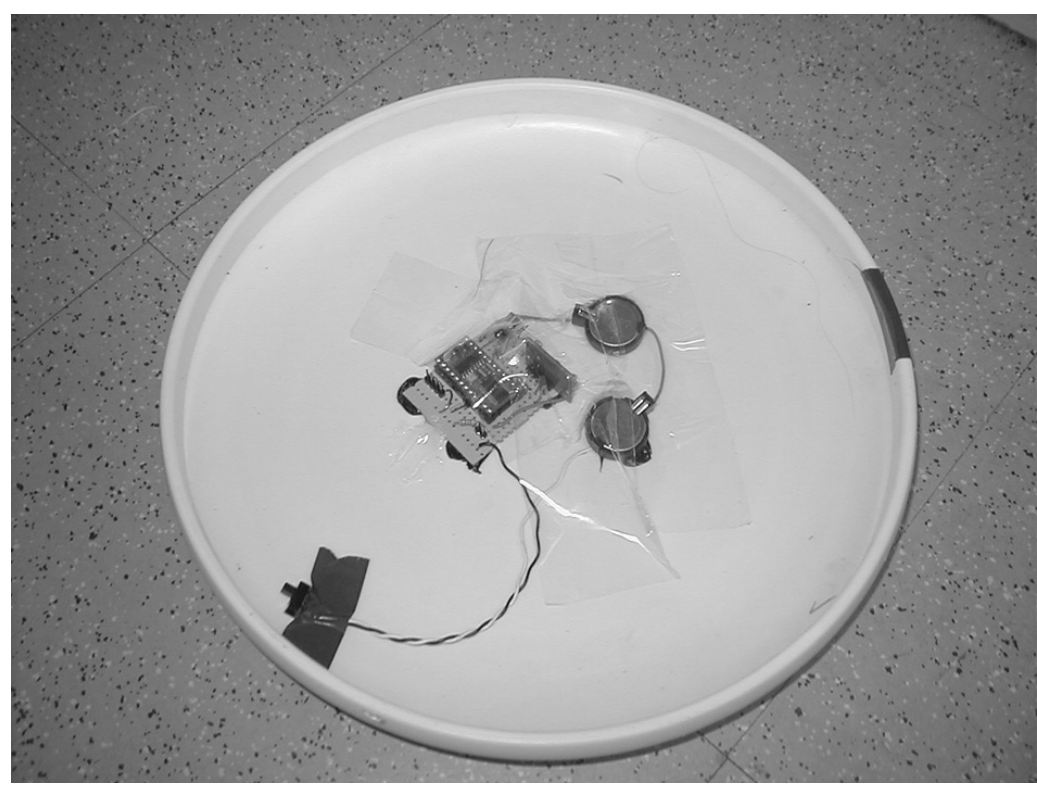

Figure 1. View of the underside of the first-generation Frisbee, $27.5 \mathrm{~cm}$ across. The microcontroller board and accelerometer are flanked by the two button cells. Wire leads to a switch near the rim of the Frisbee permit activation at the moment of launch. Clear adhesive tape fairs the equipment to minimize aerodynamic perturbations.

Huygens probe to Titan, are spun for attitude stability. Their dynamics are inferred from the data telemetered from a small number of sensors such as gyros and accelerometers to determine the vehicle's flight path, and to infer the density of the atmosphere from the aerodynamic deceleration in flight. Since the measured deceleration relates directly to both the atmospheric density and the drag coefficient, it is vital for accurate atmospheric measurements that the drag coefficients as a function of angle of attack be known, and the angle of attack history be known.

The purpose of this paper is to document a series of experiments exploring the utility of on-board sensing in freeflight models. In addition to demonstrating the feasibility of the technique for aerodynamic measurements, this paper provides examples of nonideal flight data, to aid in the interpretation of real-world sensors. An example is unsteady attitude motions such as nutation which can significantly affect the dynamics in free flight: this motion is suppressed in windtunnel tests by the rigid sting mounting. While such motions may be considered nonideal, they are quite real and have a significant effect on flight. Disc wings have also been proposed as an architecture [4] for unmanned aerial vehicles (UAVs) onboard sensing will be necessary to implement guidance and control on such vehicles.

\section{Initial experiments}

A commercial (175 g 'Wham-O Competition Frisbee') disc was obtained for tests. Initial experiments used an Analog Devices ADXL202 two-axis accelerometer, with its pulsewidth modulated digital data sampled and stored by a Parallax Inc., Basic Stamp 2 microcontroller (BS2IC). This $\sim \$ 50$ unit in a 24-pin dual in-line package is easily programmed in a high-level Basic language, but has a fairly modest current draw of around $\sim 6 \mathrm{~mA}$. This permitted the use of very small lithium 'button' cells (model CR2032) as a power source. The two accelerometer axes were mounted along and orthogonal to the axis of the disc and were calibrated simply by holding the disc with the sensitive accelerometer axes along the local and against the local gravity vector.

The microcontroller was programmed to sample the two accelerometer axes, recording each result as an 8-bit number (representing from +2 to $-2 g$ ) into its on-board EEPROM. The $2 \mathrm{~K}$ space on the microcontroller permitted about 800 pairs of samples, in addition to the program code. These samples were acquired in about $12.5 \mathrm{~s}$, yielding a data acquisition rate of about 65 sample pairs per second. We have used similar equipment to measure swing dynamics of parachuteborne instrument packages [5].

The microcontroller and accelerometer were attached with silicone adhesive to the underside of the Frisbee (see figure 1), with the accelerometer mounted as close as could be determined by eye to the centre of the disc. The batteries were similarly mounted close to the other items to minimize any displacement of the centre of mass or change in moments of inertia. A small power switch was installed near the rim of the disc, to permit turn-on as the disc was thrown.

The equipment had a mass of about $28 \mathrm{~g}$, giving a flying weight of $204 \mathrm{~g}$, about 15\% higher than the 'clean' Frisbee. Design, assembly and programming time amounted to around $8 \mathrm{~h}$ (further particulars of the construction, and code available for download, have been published in the popular electronics literature [6]). More serious weight-reduction efforts, and in particular the exclusive use of surface-mount components, could reduce the instrumentation mass by factor of $2-3$, although at the expense of considerable labour.

The instrumentation had a side area of around $3 \mathrm{~cm}^{2}$, although none of it projected below the lip of the disc. Flow disturbance was minimized by fairing the equipment with adhesive tape, to present a smooth profile. Since windtunnel tests show that the pressure on the underside of a disc is in fact quite modest and uniform, it is believed 


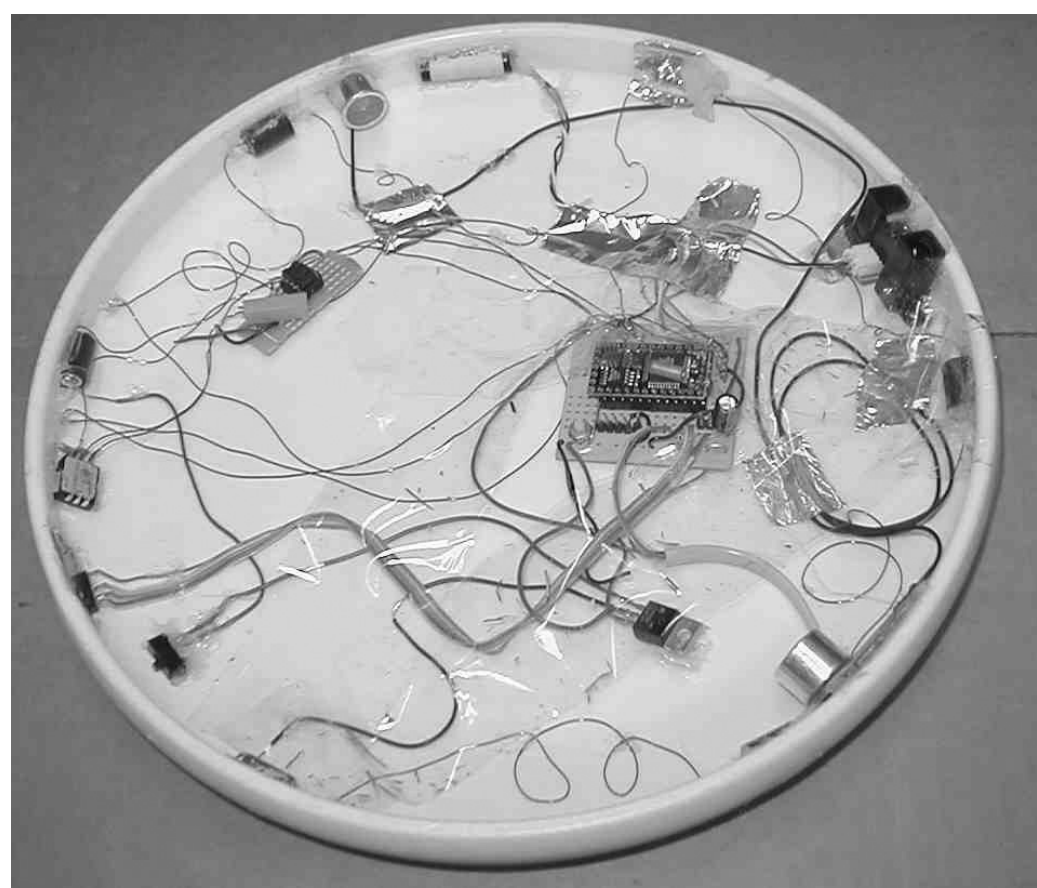

Figure 2. Second generation Frisbee. Components on rim are magnetometer (at top), infrared ranger at $\sim 2$ o'clock, pressure sensor at $\sim 9$ o'clock, flight accelerometer ( $\sim 8$ o'clock $)$ and ultrasonic time-of-flight anemometer (11.30 and 4 o'clock) and batteries $(\sim 11,9.30,7,5$, 3,1 o'clock). Other components visible closer to the centre are the microcontroller board ( $\sim 3$ o'clock), voltage regulator (5 o'clock), pressure sensor amplifier ( $\sim 10$ o'clock) and switch (7.30).

that the instrumentation's perturbation to the aerodynamic characteristics is minimal.

After the data acquisition window ended, the microcontroller read the data out at 9600 baud to a serial cable which could be attached to a laptop computer after landing. The data were captured as a text file by a terminal program for off-line analysis. These data alone, however, could not provide unambiguous recovery of aerodynamic coefficients, since the attitude or speed was not simultaneously determined. However, the exercise demonstrated that data of adequate quality can be obtained and recovered and encouraged further tests.

\section{Second and third generation tests}

A second, more elaborate set of instrumentation was developed in early 2004 (figure 2). This used a more powerful microcontroller. The Netmedia Inc., BX-24 microcontroller is a device with 32000 bytes of EEPROM for program and data storage, with eight on-board 10-bit analogue-todigital converters, also programmable in a Basic programming language.

The BX-24 required a power source with higher current than CR2032 button cells could provide. We used a string of 6 1/3-AAA nickel metal hydride cells giving 7.2 V. These cells were mounted along the inside of the rim of the disc.

Components were mounted using a glue gun, usually in holes or recesses cut with rotary tools both to maximize the security of their attachment (discs tend to be made of highdensity polyethylene or polypropylene to which adhesives do not bond well), to minimize the projection of components into the airflow, and to minimize the change in weight and moment of inertia introduced by their installation.
This second-generation disc employed ADXL202 accelerometers as before (in this case mounted on the rim of the disc, rather than projecting up from its centre). Two sun sensors were mounted, one roughly flat on the upper side of the disc, the other on a steeply angled part of the rim. The sun sensors are simple flat photodiodes, embedded in the plastic of the disc. These allow a photocurrent, roughly proportional to the cosine of the angle between the normal to the photodiode and the line to the sun, to flow from the regulated $5 \mathrm{~V}$ supply to ground through a $100 \mathrm{k}$ resistor, with the voltage across the resistor being read by an analogue-to-digital port on the BX24. During early Tucson summer, the sky is persistently clear and thus sun sensors yield excellent signals.

A small, cylindrical fluxgate magnetometer unit (FGM-1) sold for mobile robotics and industrial sensing applications was tried. This provides a $5 \mathrm{~V}$ square wave pulse with a period proportional to the field along the sensitive axis and one was mounted inside the rim of the disc with its axis oriented tangentially to the disc.

Sensors also tried on the second generation disc also include a Sharp GP12A02 infrared range sensor (this projects a spot of infrared light at an angle onto a scene, and uses a position-sensitive detector to measure the spot position and thus determine the distance to the reflecting surface). This small sensor gives an analogue voltage for distances between about $20 \mathrm{~cm}$ and $80 \mathrm{~cm}$. Although some interpretable data were obtained, the somewhat uneven sensor output and low update rate (new distances are determined only 30 times per second) eroded the sensor's usefulness.

A small sonar unit (Devantech SRF-04) was modified to transmit an ultrasound pulse across the underside of the disc, in the hope of obtaining a spin-modulated time-of-flight 
Frisbee flight and attitude dynamics

Table 1. Sensor characteristics.

\begin{tabular}{|c|c|c|c|c|c|c|}
\hline Sensor & Dimensions $(\mathrm{cm})$ & Mass (g) & Type & Current & Range & Output \\
\hline Pressure & $1.0 \times 1.0 \times 0.4^{*}$ & 2 & Piezoresistive FPX-014 & $2 \mathrm{~mA}$ & -0.25 to $1.25 \mathrm{kPa}$ & Analogue $0.25-4.25 \mathrm{~V}$ \\
\hline Magnetometer & $0.6 \mathrm{dia} \times 2.5$ long & 4 & Fluxgate FGM-1 & $8 \mathrm{~mA}$ & -50 to $+50 \mathrm{nT}$ & Square $20-80 \mathrm{kHz}$ \\
\hline Accelerometer & $0.3 \times 0.8 \times 1.5$ & 1 & Micromachined ADXL210 & $0.5 \mathrm{~mA}$ & -10 to $+10 g$ & $1 \mathrm{kHz}$ PWM \\
\hline Accelerometer & $0.3 \times 1.2 \times 1.5$ & 1 & Micromachined ADXL202 & $0.5 \mathrm{~mA}$ & -2 to $+2 g$ & $1 \mathrm{kHz}$ PWM \\
\hline Solar & & $<0.1$ & Photodiode & $\sim 1 \mathrm{~mA}$ & $0-1350 \mathrm{~W} \mathrm{~m}^{-2}$ & Analogue $0-4 \mathrm{~V}$ \\
\hline IR ranger & & 4 & IR spot GPD12 & $30 \mathrm{~mA}$ & 0.2 to $2 \mathrm{~m}$ & Analogue $0-2.5 \mathrm{~V}$ \\
\hline Sound speed & $2.5 \times 1.5 \times 1$ & 6 & 40 kHz SRF04 & $30 \mathrm{~mA}$ & & Square pulse \\
\hline Sonar ranger & $2.5 \times 1.5 \times 1$ & 6 & 40 kHz SRF08 & $30 \mathrm{~mA}$ & 0 to $6 \mathrm{~m}$ & Serial integer \\
\hline Microphone & & $<1$ & Electret & $\sim 2 \mathrm{~mA}$ & & Pulse rate \\
\hline
\end{tabular}

* Sensor body only—pressure part extends a further $8 \mathrm{~mm}$.

measurement that might therefore indicate the mean airspeed across the underside of the disc. This was generally noisy, although it did sometimes yield a perceptibly spin-modulated signal.

Finally, a Fujiwara XP150 pressure sensor was mounted on the curved rim. This is a differential pressure sensor with built-in temperature compensation and amplification giving a $0-4.25 \mathrm{~V}$ output range for pressure differences of $150 \mathrm{~Pa}$. For this application, the sensor output was amplified by a further factor of 30 in the hope of measuring directly the suction peak on the upper leading edge of the disc. A differential measurement is somewhat unsatisfactory in that the 'reference' pressure is that inside the lip on the underside of the disc, which itself changes during the rotation. Again, some notably spin-modulated signals were recovered, but not reliably.

This second generation testbed confirmed that the attitude sensors and the BX24 and battery installation were workable, even though they brought the instrumented disc mass up to $260 \mathrm{~g}$. The large number of sensors, confronted with the fixed sampling throughput of the microcontroller, gave sampling rates slightly lower than ideal.

Thus the platform was modified into the third generation disc, with which the principal results of this paper were obtained. This used fundamentally the same architecture, except with a second BX24 microcontroller running in parallel to double the data acquisition capability. A second FGM-1 magnetometer was mounted radially, canted at $45^{\circ}$ to the disc axis (it was too long to be mounted along the disc axis without projecting into the airflow.), as well as a second accelerometer. This two-axis device, an ADXL210, was mounted flat on the disc centre. This device has a sensitivity somewhat low for flight measurements (it is identical to the ADXL202 but with a range of $\pm 10 \mathrm{~g}$ ) but permits the recording of the accelerations during the throw.

The acoustic anemometer was replaced with a downlooking sonar used as an acoustic altimeter. The device itself (SRF-08) is the same as the SRF-04 used in the time-offlight experiment, but with the incorporation of a dedicated microcontroller to perform timing. This allows the master processor (the BX24) to perform other duties during the altimetry measurement. Whereas the time-of-flight pulse takes under a millisecond to cross the disc, in the altimetry application the waiting time would be prohibitive - at typical heights of $3 \mathrm{~m}$, the sound pulse takes some $18 \mathrm{~ms}$ to return.

A final experimental sensor was tried. This simply placed an electret audio microphone (Jameco Electronics, $55 \mathrm{~dB}$ sensitivity) element on the upper surface of the disc, with a $\times 200$ gain amplifier. This large gain tends, by design, to saturate the amplifier if air is blown across the microphone (much as one will commonly test a public address system by blowing on the mike) to provide a train of digital pulses: the number of pulses is counted in a $2 \mathrm{~ms}$ period and stored.

Sensor parameters are summarized in table 1.

\section{Sensor calibration}

Some analogue signal processing was performed in hardware (e.g., resistor network on the sun sensors, amplifier for the pressure sensor). Further processing was performed in software to yield an 8-bit number for each sensor to facilitate storage as a single byte.

Sensor calibration was performed as before for the accelerometers. The microphone was a purely experimental sensor and was not given an independent quantitative calibration-it may respond more to a turbulent flow rather more than a steady flow at the same speed. Since the pressure sensors provided no interpretable data, no independent calibration was performed on them.

The attitude sensors (sun sensors and magnetometers) were calibrated by mounting the Frisbee on a motorized altazimuth mount (that of a Meade LX200 $20 \mathrm{~cm}$ telescope). Although the orientations were simply set manually with the motors turned off, this approach yielded a very stable, smooth mount for making measurements at a selection of attitudes. After aligning level and due north, the calibration attitudes were simply read off from the telescope setting circles. A 'lazy susan', a bearing table for presenting condiments in a kitchen, was attached to the mount and could be spun by hand.

Results are shown in figure 3 . The signal processing was designed to yield a reading with an approximately cosine relationship to the angle between the sun (or field) direction and the sensor axis. Between the peak and the trough of the reading, the orientation measurements may have a sensitivity of around $1 \mathrm{DN}$ per degree (all measurements are recorded and reported as 8-bit integer data numbers (DN)).

\section{Range instrumentation}

To determine aerodynamic coefficients, the flight conditions (specifically flight speed and flight path angle) must be known. These are difficult to obtain with on-board measurements, since GPS measurements are not practicable on such short flights, and so additional instrumentation is needed.

A conventional video camera was set up in the test field. The record from this camcorder was digitized after the tests, 


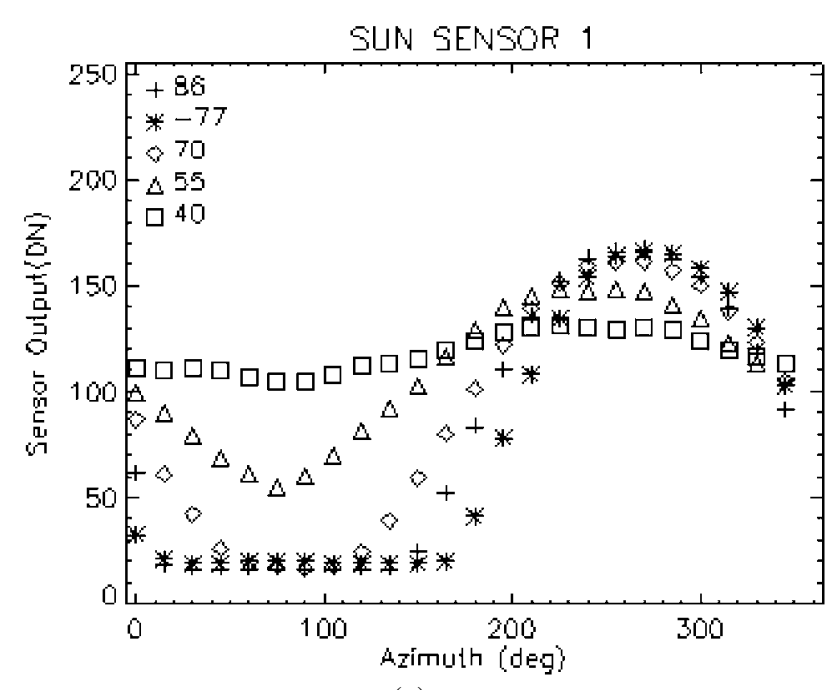

(a)

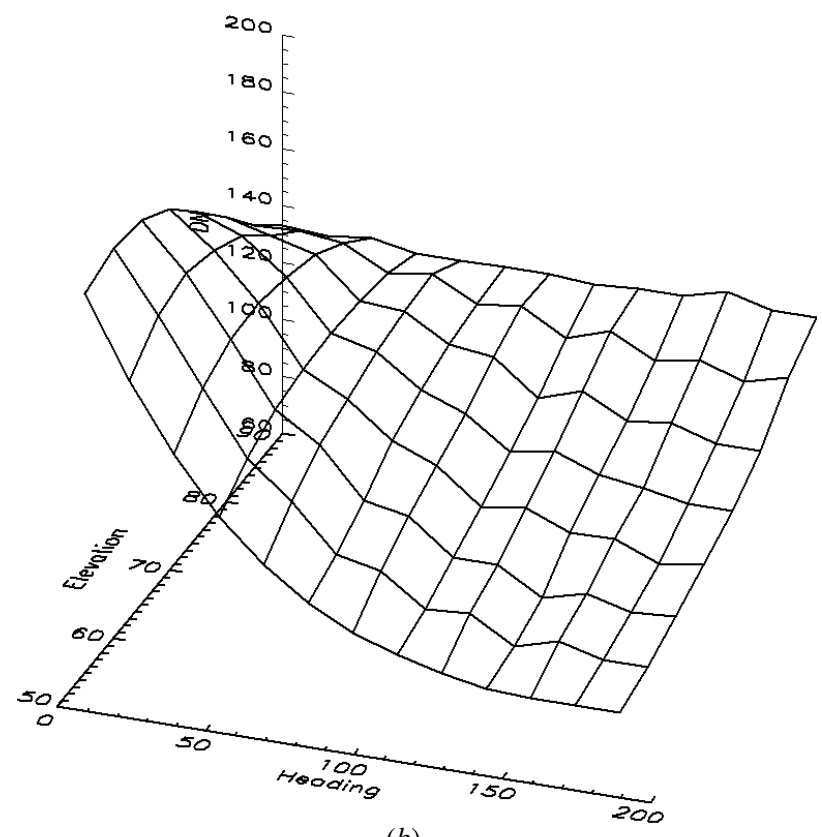

(b)

Figure 3. Example sensor calibration data. (a) Shows sun sensor 1 output, with the disc attached to the telescope mount and not rotating. Various symbols correspond to different elevation angles (90 degrees is vertical) and the telescope (and spin axis) is rotated to different azimuths. (b) Carpet plot of the minimum magnetometer 1 output as the disc spins with the spin axis at various elevations and spin axis azimuths.

and $X-Y$ positions of the disc in the image plane were recorded for each frame to yield a record of the translational motion of the disc for most of its flight, in particular providing a launch speed and angle constraint. The image coordinates were converted into the physical distance using red cones placed in the field as fiducial markers every $2.5 \mathrm{~m}$. For full 3-D trajectory recovery, two video cameras would be used, but since the disc was always thrown in a known direction relative to the line of sight-usually within $30^{\circ}$ of the same (due east) azimuth - the speed can be corrected by a cosine factor.

We also experimented with a radar 'speed gun'. This instrument, a hand-held microwave Doppler device which measures the velocity radial to it, is sold for sporting applications (e.g., baseball pitches), but was not found to be useful - it takes a second or so between activation and report, during which time the disc changes speed, and line of sight direction, significantly. Thus these data were not judged to be reliable compared with the video record.

Note that an independent measurement of the launch speed can be obtained by studying the launch acceleration record, captured by the ADXL210 and magnetometers. Similarly, knowing the flight distance and time from launch to impact, constraints on the flight speed can be imposed from which realistic speed histories can be constructed. However, it is more robust and accurate to obtain an independent velocity measurement. Video digitization is somewhat laborious-we may in future experiment with ultrasonic or infrared beacon navigation.

In passing we note the related work of Hummel [7, 8], who has conducted free-flight measurements using a dedicated high-speed video system (120 and 200 frames per second). Three light emitting diodes were mounted on the Frisbee and their positions recorded in flight.

From position data alone, one can either compute rates and accelerations by differentiation (which can be susceptible to noise) and thereby compute instantaneous aerodynamic coefficients, or use smooth analytic functions to describe the trajectory itself and differentiate those numerically or analytically to calculate instantaneous coefficients, or use smooth analytic functions to describe the coefficients themselves, forward model the resultant trajectory and tune those functions to fit the position data. This latter approach was taken by Hummel and has been used in ballistic range tests of planetary entry probes.

\section{Flight results}

The video trajectory data for one flight (no 4) are shown in figure 4. The characteristic 'airfoil-shaped' trajectory is observed, with a near-linear shallow upwards ramp before the disc has slowed appreciably, then a slow and steepening descent. The video record was obtained looking north, while the disc was thrown in an ESE direction. From the perspective of the thrower (not apparent in this video record) the disc curved slightly to the left (north) towards the end of its flight. The eastward distance was $22 \mathrm{~m}$, with a $\sim 6 \mathrm{~m}$ slide to the $\mathrm{N}$.

The velocities obtained by position differences in the video record have some scatter, but broadly linear trends are evident in both horizontal (i.e., E) and vertical velocities. A faint indication is seen in the vertical speed record of an increase in the vertical velocity immediately after the throwthe lift accelerates the disc upwards.

Figure 5 shows the attitude sensor and accelerometer data from the same throw. Spin modulation is seen on both magnetometers and sun sensors at $\sim 6.5 \mathrm{~Hz}$. Modulation envelope varies due to the slow precession of the spin axis during flight.

The accelerometers are over-ranged (span is $\pm 2 g$ ) at launch and impact. The radial accelerometer is spin modulated as expected about its zero value of $170 \mathrm{DN}$, though with some twice-per-rotation nutation signal just after launch. Hummel [8] has similarly observed nutation in the first second or 

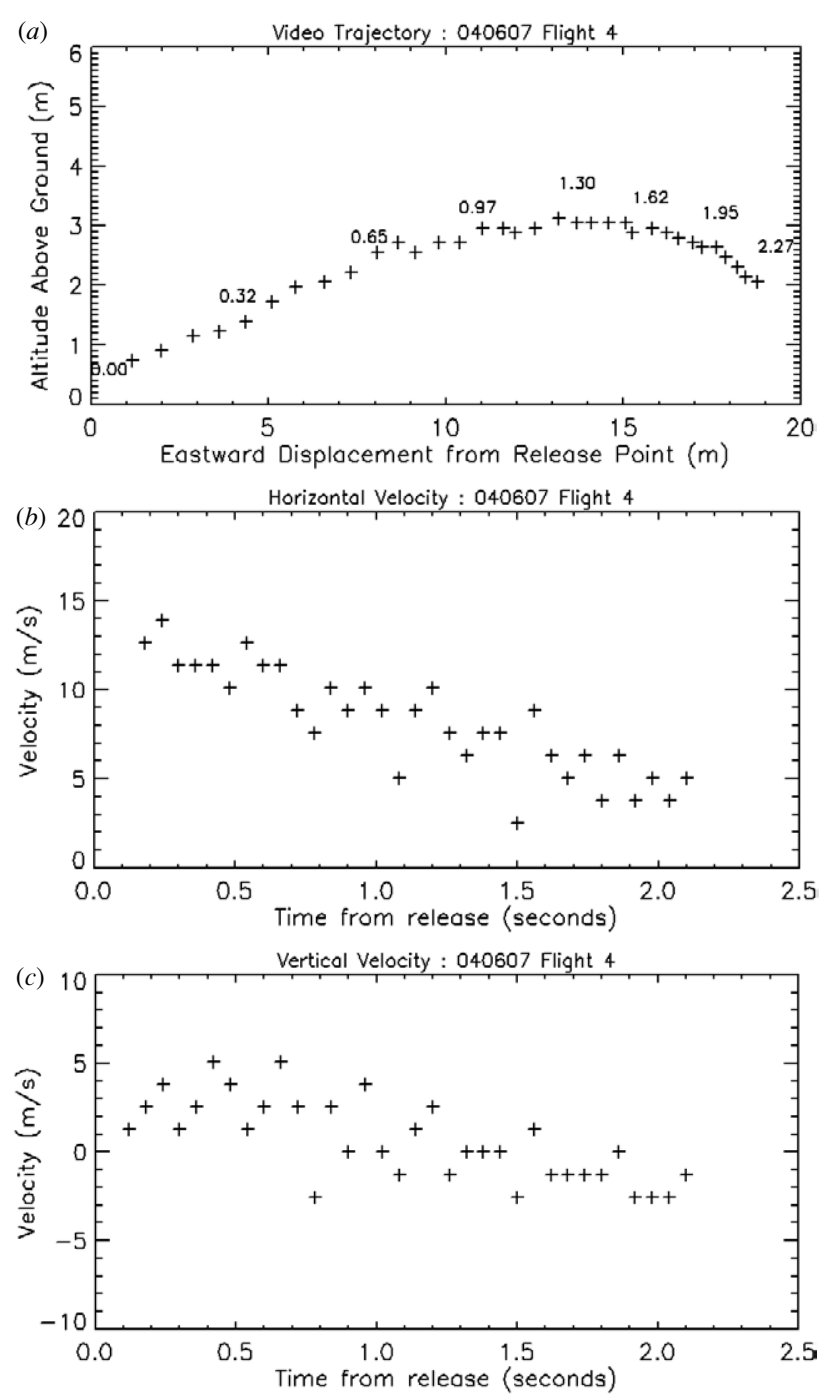

Figure 4. Frisbee trajectory from video record. (a) $X-Y$ position, with time in seconds indicated along the trajectory. (b) Horizontal velocity component as a function of time- the disc slows rather uniformly, at around $6 \mathrm{~m} \mathrm{~s}^{-2}$. (c) Vertical velocity as a function of time-also decreasing approximately linearly, this time at $3-4 \mathrm{~m} \mathrm{~s}^{-2}$. The flight path angle decreases from about $+15^{\circ}$ upwards to $-30^{\circ}$ before the Frisbee left the video frame.

so of Frisbee flight - the nutation is caused by the angular momentum vector imparted by the thrower being misaligned with the axis of maximum moment of inertia. Energy dissipation, either by flexing of the disc or by aerodynamic forces, tends to damp this nutation quickly. The axial accelerometer shows spin modulation (coning) due to sensor misalignment with principal axis, about a near-constant flight signal of $\sim 1.3 g$.

Figure 6 zooms in on these parts of the acceleration signals, with a magnetometer overlain to indicate the spin phase. The accelerometers are particularly sensitive to nutation and coning effects, which are well known in spacecraft engineering. Nutation is commonly observed in spinning satellite deployments due to slight tip-off errors on separation-Spencer et al [9] show accelerometer data from the Mars Pathfinder entry probe with a nutation signal (although they incorrectly label it as 'coning'), and a nutation
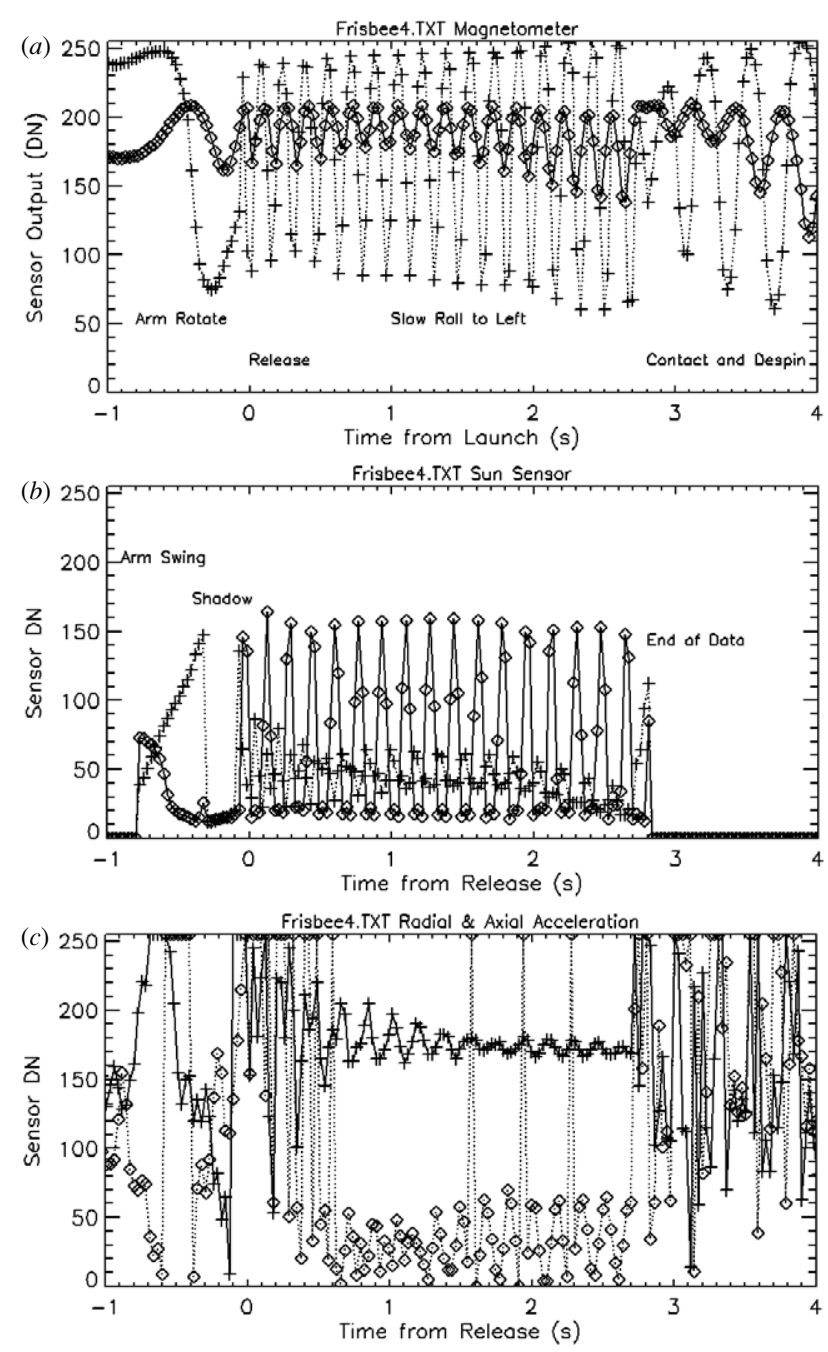

Figure 5. Data from dynamics sensors—same flight as figure 4 . Magnetometer record $(a)$ shows spin modulation on magnetometers 1 (dotted line/crosses) and 2 (solid line/diamonds) at $\sim 6.5 \mathrm{~Hz}$. Modulation envelope varies due to tilt of spin axis during flight. Sun sensors $(b)$ show similar effects. Accelerometers (c) are over-ranged at launch and impact. Radial accelerometer (solid line/crosses) is spin modulated about its zero of $170 \mathrm{DN}$, though with some twice-per-rotation nutation signal just after launch. Axial sensor (dotted line/diamonds, with zero level 110) shows spin modulation (coning) due to sensor misalignment with principal axis, about a near-constant flight signal of $\sim 0.6 g$.

was generated on the Giotto spacecraft [10] by a dust impact during its close approach to comet Halley in 1986.

Figure 7 shows the record from the microphone sensor on the upper surface near the rim of the disc. This too shows a spin modulation, as the microphone sweeps round from leading to trailing side, suggesting it responds to the airflow immediately adjacent to the disc surface. The modulation varies somewhat throughout the flight due to the variation in flight speed and the angle of attack. Future work will investigate the utility of this type of sensor further.

\section{Attitude reconstruction and dynamics}

The determination of the spin axis and phase of a rotating vehicle is of course a standard problem in spacecraft attitude 

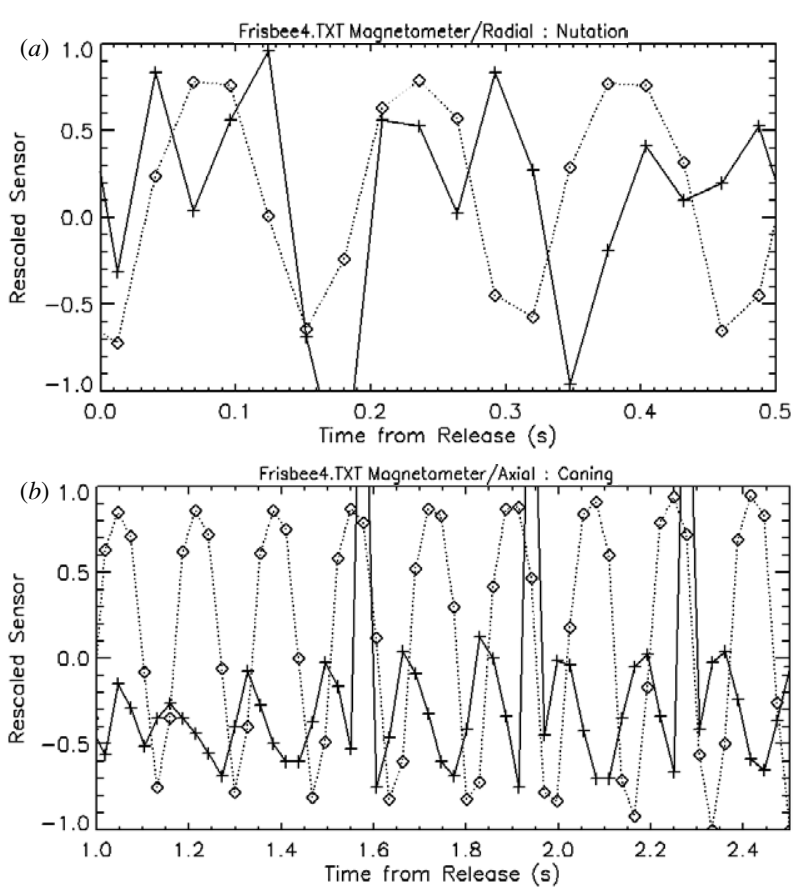

Figure 6. Nonideal dynamic signatures in the acceleration records (solid line/crosses), with magnetometer for spin-phase reference (dashed line/diamonds). (a) Shows a nutation signature ( $\sim$ twice per spin period) in the radial acceleration early in the flight-compare with figure 5 later in the flight where this signature is damped out. (b) Shows coning (once per spin period) in the axial accelerometer, indicating that the accelerometer is not truly aligned with the spin axis. The axial accelerometer also shows nutation early in the flight.

dynamics, and a combination of sun sensor and magnetometer is often used (e.g. [11, 12]). A major difference here is that the spin axis of a Frisbee in flight is being precessed rapidly, whereas the generally very small disturbance torques in space mean a spacecraft is likely to maintain a constant spin axis and rate over periods of weeks, and a variety of filtering approaches can be used to estimate the attitude. As one example, ground software used sensors on the DARPASAT satellite [12] spinning at $20 \mathrm{rpm}$ to generate attitude estimates of better than $1^{\circ}$ from 2 min of data, i.e. 40 spin periods. The discs here can only be considered to have a pseudoconstant attitude for around five spin periods. (It may be noted in passing that sensor data on DARPASAT, a vehicle three-four orders of magnitude higher in cost than the instrumented disc, also show nutation and coning signals.)

Full exploitation of the data from Frisbee flights with a fixed (rather than evolving and experimental) sensor configuration may be best accomplished with a forward model of the flight and attitude dynamics, which computes explicitly the expected signal from each of the sensors. By performing Euler rotations, the dot product of the magnetometers with the field vector, and that of the sun sensors with the solar vector, can be calculated at every instant of flight, and a sensor model applied to derive the corresponding stored data number. Expected acceleration signals can similarly be computed. Launch conditions and a model of the aerodynamic coefficients can be adjusted such that the model time series of the various sensors match those recorded.

For the present proof of concept where continual improvements are being made to the hardware and software configuration, we employ a heuristic approach, wherein the envelope of the spin-modulated sensor data is used to define, by manual best fit to the data such as that in figure 3, the spin axis direction. Magnetometer 1 minimum and maximum signals, magnetometer 2 minimum and the sun sensor maxima were used.

In this exercise, tests were performed quite deliberately in the morning around 8 am, when the sun was sufficiently high above the horizon to give a good signal, but was still well in the east. Early afternoon tests would have suffered not only from more convectively unstable conditions with stronger winds, but the sun direction and magnetic field direction would then be approximately collinear, making the attitude determination degenerate.

Since mechanical construction was performed with hand tools and adhesives, without precision alignment fixtures, sensor orientation was determined by post-hoc measurement. As an example, sun sensor 2 was nominally mounted flat in the plane of the disc but after the adhesive set was found to have a perceptible, but difficult to measure, inclination to the plane.

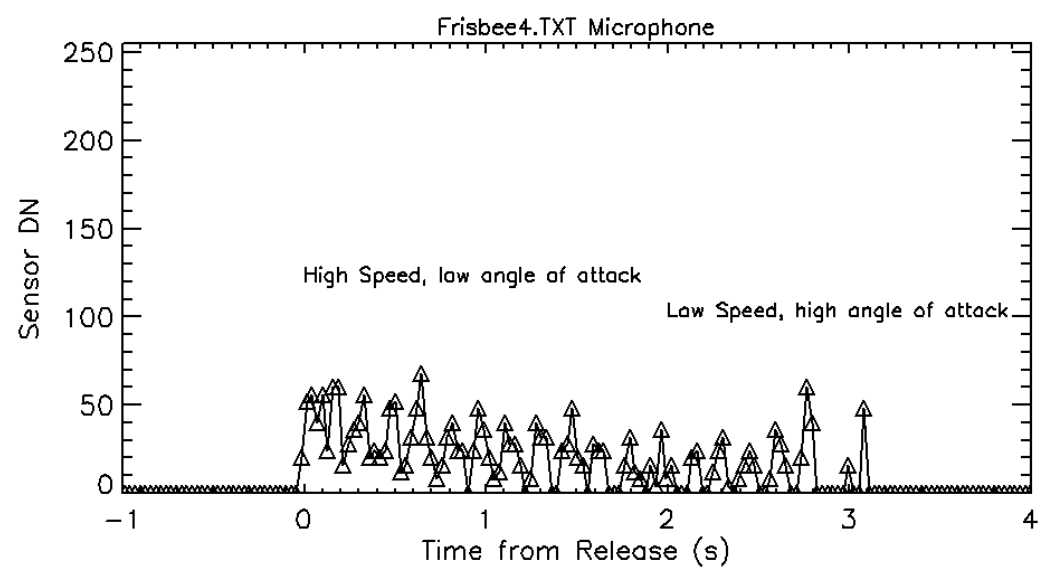

Figure 7. Upper surface microphone output (pulses per $4 \mathrm{~ms}$ ). Both maximum and minimum values in each spin period decline from launch, indicating a probable speed dependence. Maximum value appears to increase during the last second of flight, suggesting perhaps an angle-of-attack dependence. 

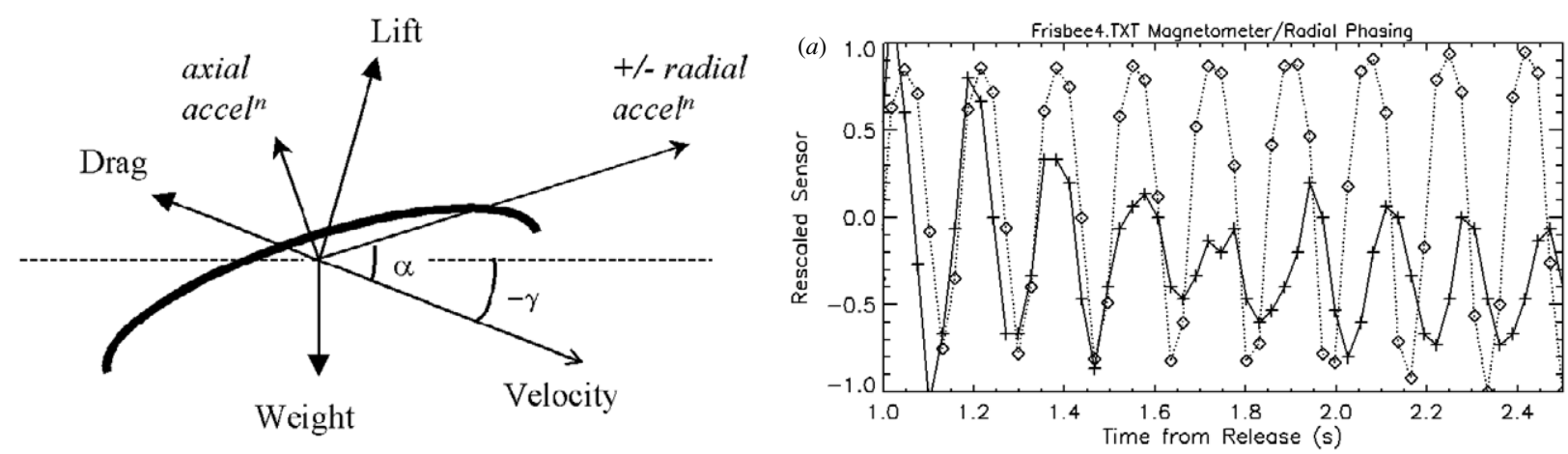

\section{$\alpha=$ Angle of Attack \\ $\gamma=$ Flight Path Angle}

Figure 8. Forces on flying disc, and their measurement. Measurements are body fixed, ideally along the spin axis and orthogonal to it (i.e. radial). Sensors measure body attitude with reference to horizontal (dashed line). Lift and drag forces are defined with reference to the velocity relative to the air. Flight path angle (positive upwards) is angle between velocity and horizontal; angle of attack is angle between body axis and velocity. Note that in the body frame at this attitude, lift and drag have components in opposite radial directions.

Modest spin modulation is therefore evident in its flight data (e.g. at one point in flight 6, the reading varies 20-45; later the variation is 25-70). Both of these ranges (other data show the sensor to report approximately $20+130 \cos (\Theta)$ where $\Theta$ is the angle between the sensor normal and the sun direction) are consistent with a mounting offset of $6^{\circ}$.

For the flight shown in figures 4 and 5 , the sensor data around $1 \mathrm{~s}$ after release were best fitted with an elevation of $70^{\circ}$ (i.e., spin axis was $20^{\circ}$ from vertical) and an azimuth of $120^{\circ}$ (i.e., the spin axis was tilted towards WNW). If the disc was flying horizontally in an ESE direction, it would therefore have a $20^{\circ}$ angle of attack (see figure 8 for the relationship of the body sensor axes to lift and drag, and the definition of angle of attack and flight path angle). The estimated accuracies for heading and elevation are $15^{\circ}$ and $5^{\circ}$, respectively-it was noted that it was difficult to obtain an attitude solution that agreed with all of the sensor outputs simultaneously. One possibility is that the magnetometers have a slight temperature dependence, leading to an offset in readings from the calibration setup to the flight test.

At $\sim 2.5 \mathrm{~s}$, shortly before impact, the attitude has precessed due to the disc's pitch moment. The best-fit attitude has an elevation of $60^{\circ}$ and a heading of $150^{\circ}$. This corresponds to a precession of about $14^{\circ}$ in total. Inspection of the flight data suggests that this change occurred principally between $1.5 \mathrm{~s}$ and $2.5 \mathrm{~s}$ into the flight, and there was a slight change in the opposite direction immediately after release.

Note that moment coefficients can only be derived by measuring the change in attitude over some section of the flight, since (unlike the lift and drag, which are recorded instantaneously by the accelerometers) the attitude rates are not measured directly.

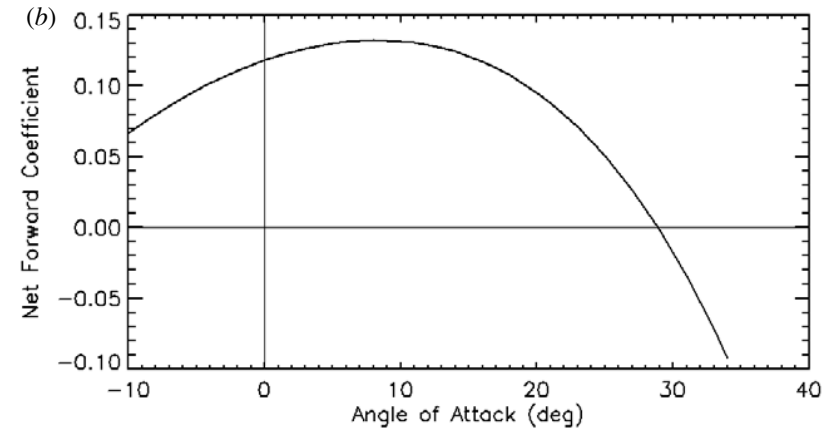

Figure 9. (a) The rescaled radial acceleration record (solid line/ crosses) against the magnetometer record (dotted line/diamonds). Note that the acceleration initially peaks before the magnetometer, a phasing that could be used to determine the direction of flight. Within four spin periods $(<1 \mathrm{~s})$ the phasing becomes reversed, due to the swiftly increasing angle of attack, and thus the increasing radial component of lift. (b) The net forward radial component of lift and drag, $C_{\mathrm{d}} \cos (\alpha)-C_{1} \sin (\alpha)$, using a quadratic and linear fit to $C_{\mathrm{d}}$ and $C_{1}$ (see figure 10 ): at just under $30^{\circ}$, the net component is zero. Beyond $30^{\circ}$, the component is negative, and hence the phase of measured radial acceleration with respect to the forward direction is reversed.

\section{Discussion}

\subsection{Accelerations}

The acceleration recorded by the radial accelerometer is by design spin modulated. The mean value is nominally zero, although sensor positioning and orientation may introduce a centripetal acceleration component, with an amplitude related to the near-constant spin rate. While the modulation envelope is defined by the lift and drag forces, the phase of the peaks and troughs in the signal relative to the peaks and troughs in the attitude sensor record can be used to infer the projection of the flight direction in the spin plane, a useful parameter in guiding a disc wing via spin-phased actuators such as flaps.

Some care is needed, however, since the radial signal amplitude and phase are affected not only by the magnitude of the lift and drag, but also by the attitude (see figure 8) which controls how these two forces are projected into the spin plane and thus the radial sensing direction.

Figure 9 shows the radial accelerometer record towards the end of flight, with magnetometer 1 overlain as a phase reference. It can be seen that the acceleration peaks before the magnetometer, indicating a particular heading. However, within four spin periods $(\sim 0.6 \mathrm{~s})$ the phase reverses, with the acceleration peaking after the magnetometer. This does not indicate a $180^{\circ}$ turn in flight, but rather the changing 
dominance of the two terms that contribute to the radial acceleration $(D \cos (\alpha)-L \sin (\alpha)) / M$, where $M$ is the mass of the disc $(0.26 \mathrm{~kg})$ and other terms are defined in figure 8 . Using a linear fit $\left(C_{1}=0.2045+0.0495 \alpha\right)$ to the lift coefficient data of Potts and Crowther [3], and a parabolic fit to the drag coefficient, namely $C_{\mathrm{d}}=0.118+0.0069 \alpha+0.0007 \alpha^{2}$, the net radial force coefficient $C_{\mathrm{d}} \cos (\alpha)-C_{1} \sin (\alpha)$ can be calculated, and is shown in figure $9(b)$.

It is seen that this function has a maximum value at a modest angle of attack $\left(\sim 8^{\circ}\right.$, coincidentally close to the angle at which a disc has zero pitch moment, i.e. flies in trim). Between $20^{\circ}$ and $35^{\circ}$, the function decreases rapidly and becomes negative. It is this change of sign that is responsible for the change in phase of the radial acceleration signal.

The function is zero, corresponding to a vanishing of the radial signal, at a critical angle of attack of around $28^{\circ}$. In principle, one can deduce from the radial acceleration record when the disc encounters this critical angle (though of course the value will depend on the actual variation of the coefficients with angle of attack - the plot shown is from only the linear and parabolic fits).

\subsection{Aerodynamic coefficients}

Knowing the body attitude and the velocity of flight (both speed and direction), the lift and drag coefficients can be calculated from the instantaneous accelerations (side force is assumed to be zero). Additional parameters are disc mass and area $\left(0.26 \mathrm{~kg}\right.$ and $\left.0.057 \mathrm{~m}^{2}\right)$, air density $\left(\sim 1.1 \mathrm{~kg} \mathrm{~m}^{-3}\right.$ at Tucson altitude of $780 \mathrm{~m}$ ).

Data points are shown in figure 10 for coefficients computed 1 and $1.5 \mathrm{~s}$ into the flight, when the horizontal velocities were $\sim 9$ and $7 \mathrm{~m} \mathrm{~s}^{-1}$, and flight path angles $\sim 12^{\circ}$ and $\sim 0^{\circ}$, respectively. Corresponding angles of attack (taking the measured attitude into account) are $8^{\circ}$ and $15^{\circ}$. Axial acceleration was $\sim 0.6 \mathrm{~g}$ in each case, and radial accelerations $\pm 0.25 g$ and $\pm 0.12 g$. Calculations for later in this flight were avoided, since the northward acceleration introduced progressively larger uncertainties in the angle of attack.

The moment coefficient cannot be computed instantaneously, since the body rates are not known. Instead the precession rate is derived from the attitude history - the spin axis is precessed by $14^{\circ}$ between 1.5 and $2.5 \mathrm{~s}$. Knowing the moment of inertia $\left(0.0004 \mathrm{~kg} \mathrm{~m}^{2}\right)$, disc diameter $(0.26 \mathrm{~m})$ and spin rate $\left(37\right.$ radians $\left.^{-1}\right)$, the moment coefficient over the 1.5-2.5 s interval when the angle of attack was around $20^{\circ}$ is found to be $\sim 0.02$. The first second of flight suggests the coefficient is of opposite sign and around two to three times smaller when the angle of attack is $\sim 8^{\circ}$.

In general the agreement with previous results is quite good, perhaps closer to the wind-tunnel data of [3] than video data of $[7,8]$. The most discrepant point is the low-incidence drag coefficient, which is rather higher in this experiment than in other reports: this may be due to drag augmentation by the sensor equipment on the underside of the disc. At higher angles of attack, the disc drag becomes larger and this effect is proportionally less significant.

Inspection of the results, and consideration of the coefficient derivations, suggests that refinement of the attitude knowledge from the present $\sim 5^{\circ}$ accuracy is more useful than
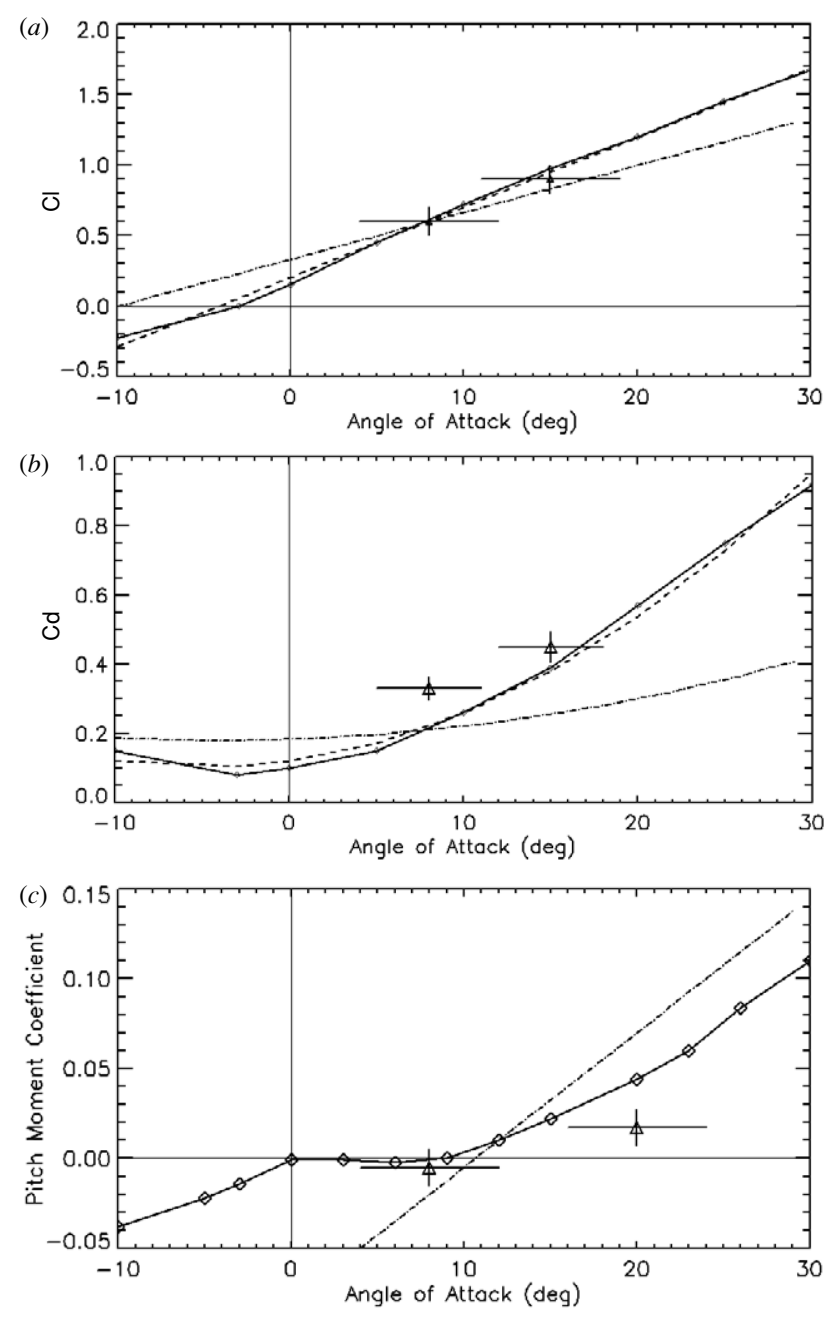

Figure 10. Aerodynamic coefficients $(a)$ lift, $(b)$ drag and $(c)$ pitch moment coefficient as a function of angle of attack. Solid curve with diamonds are wind-tunnel data from Potts and Crowther. Dashed lines are the linear and parabolic fits used in figure 9. Dash-dot lines are functions from Hummel [8] from video data. Triangles denote measurements from free flight data using the methods in the text: bars denote estimated uncertainties in coefficient and angle of attack.

improving the acceleration measurement accuracy. For later parts of the flight, the north-south motion of the disc, though not intrinsically large, becomes a significant source of error in the angle of attack.

\subsection{Launch kinematics}

A zoom of the dynamics record from the magnetometers and the ADXL210 sensors during the same throw is shown in figure 11. (Some sun sensor data were also obtained, but the thrower's body often cast shadows on the disc, making these data less useful.) It is seen that the acceleration and rotation begins smoothly, accelerating to a plateau of $\sim 2 g$ for $0.5 \mathrm{~s}$. There then follows a sharp increase in acceleration to close to $10 \mathrm{~g}$ for about $0.1 \mathrm{~s}$. Thus roughly half of the launch speed in this throw is generated in the last tenth of a second before release. It is also evident that the disc spin is almost entirely generated during this phase. 

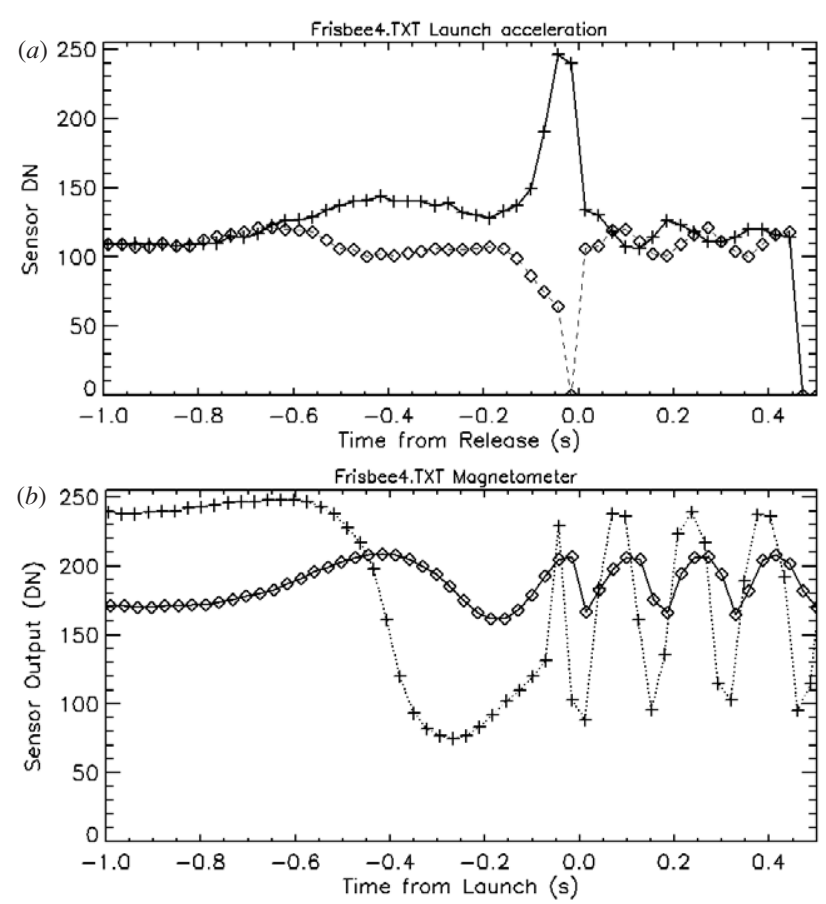

Figure 11. The two in-plane (radial) accelerations and the magnetometer record during the launch. The throw action takes about $0.5 \mathrm{~s}$ during which accelerations reach around $2 g$, while in the last $0.1 \mathrm{~s}$ accelerations (which include a centripetal component that does not contribute to the release speed) reach $\sim 10 g$. Thus roughly half of the launch speed, and almost all of the rotation, is generated in the last tenth of a second before release.

Hummel [8] has analysed the biomechanics of Frisbee throws using high-speed video and a numerical biomechanical model of the thrower. Her work also indicates most of the kinetic energy is supplied to the disc in the last $\sim 0.1 \mathrm{~s}$, by horizontal adduction of the shoulder.

\section{Conclusions and future work}

This work has demonstrated that useful flight data on Frisbee dynamics can be obtained with onboard instrumentation at rather modest effort and expense. Additional range instrumentation is required to document the flight velocity in order to recover aerodynamic coefficients: a single conventional video camera has proven adequate for the first part of flight, although a second orthogonal camera would be useful to constrain sideways motions.

A combination of magnetometer and sun sensor data can adequately determine the attitude of the disc in flight. Sensor mounting poses some tradeoffs. For spin-axis determination alone, e.g. for aerodynamic coefficient determination, data analysis would be simplified by precisely spin-axis aligned sensors. However, precise spin-axis alignment is difficult, especially since the equipment modifies the mass properties of the disc. On the other hand, sensors mounted in the spin plane can provide spin-phase information, e.g. for actuator triggering. It has been easiest in our experiments so far to mount sensors at an intermediate orientation, and recover the attitude by empirical fitting of sensor readings to data from known orientations.
Accelerometer data have generally been of good quality, although care in sensor mounting position and orientation can improve the signal by suppressing coning and nutation sensitivity. Nonetheless, relatively simple algorithms can determine when a sensor is pointed in the forward direction during steady flight-allowing the actuation of control surfaces at a specific spin phase for manoeuvring. Similarly, the acceleration peaks relative to the attitude references can permit on-board determination of heading. A combination of these two may permit autonomous guided flight of disc wings. An interesting recreational possibility might be a Frisbee that homes in on, or attempts to avoid, a person.

A bottleneck in data acquisition with the microcontrollers used here is the write-time overhead $(\sim 6 \mathrm{~ms})$ in storing data on the EEPROM. This limits data acquisition to around 200 bytes per second. With spin rates of 5-10 revolutions per second, around 40 samples per second are needed to adequately characterize the disc's behaviour, and thus not all the channels can be sampled this fast. While the write time is therefore prohibitive, data can be sampled and processed on-board much faster: for many of the measurements, it may be enough to record the short-term mean and the amplitude of oscillations about the mean.

Combined attitude information, accelerations and speed documentation from the video record, allow recovery of aerodynamic coefficients. Our measurements of lift, drag and pitch moment coefficients are in agreement with published wind-tunnel measurements. The free-flight technique lends itself to application in conditions (e.g. high$\alpha$ ) that are challenging for spinning measurements in wind tunnels.

The ultrasonic sensors (speed of sound and range) have not given encouraging results to date, perhaps as a result of turbulent conditions around the transducers. The infrared ranger gave similarly erratic results, perhaps largely due to its modest refresh rate $(\sim 25 \mathrm{~Hz})$ or poor performance in very bright conditions.

The response of the microphone sensor, with a pronounced spin-phase dependence, suggests it may be useful in measuring the flow over the disc in spinning flight. A rake of such microphones may be able to determine, for example, the effect of spin rate on the separation of the airflow on the upper surface near the stall point. Similar measurements, using pressure sensors, may allow determination of the pressure distribution on the spinning disc (wind-tunnel measurements to date have only used nonspinning discs). Our failure to acquire reliable pressure data is believed to be the result of poor sensor/amplifier implementation to date, rather than a problem with the measurement principle. The exploration of the fluid dynamics of disc-wing flight with on-board microphones, pressure sensors and perhaps hot-wire anemometers will be the subject of future work.

\section{Acknowledgments}

Jessica Dooley is thanked for assistance with these experiments, in particular for digitizing the video record. The work here was partly supported by the NASA Cassini Project. 


\section{References}

[1] Bloomfield L A 1999 The flight of the Frisbee Sci. Am. 132 (April)

[2] Lorenz R D 2004 Flying saucers New Sci. 40-1 (19 June)

[3] Potts J R and Crowther W J 2002 Frisbee aerodynamics AIAA-2002-3150 20th AIAA Applied Aerodynamics Conference and Exhibit (St Louis, MO, 24-26 June)

[4] Potts J R and Crowther W J 2002 Disc-wing UAV: a feasibility study in aerodynamics and control CEAS Aerospace Aerodynamics Research Conf. (Cambridge)

[5] Dooley J M and Lorenz R D 2003 A miniature probe-parachute dynamics testbed Proc. Workshop on Planetary Entry and Descent Trajectory Reconstruction and Science (Lisbon, Portugal, October 2003) (Noordwjik: European Space Agency) (ESA SP-544)

[6] Lorenz R D 2004 Frisbee black box Nuts and Volts vol 25 No 2 (February 2004) pp 52-5
[7] Hummel S and Hubbard M 2002 Identification of Frisbee aerodynamic coefficients using flight data 4th Int. Conf. on the Engineering of Sport (Kyoto, Japan, September 2002)

[8] Hummel S 2003 Frisbee flight simulation and throw biomechanics MS Thesis UC Davis

[9] Spencer D, Blanchard R C, Braun R D, Kallemeyne P H and Thurman S W 1999 Mars Pathfinder entry, descent and landing reconstruction J. Spacecr. Rockets $\mathbf{3 6}$ 357-66

[10] Paetzold M, Bird M K and Volland H 1991 GIOTTO-Halley encounter-when was the large nutation generated? Astron. Astrophys. 244 L17-20

[11] Santoni F and Bolotti F 2000 Attitude determination of small spinning spacecraft using three axis magnetometer and solar panels data IEEE Aerospace Conf. (Big Sky, MT, March 2000) paper 2.0203

[12] Mitchell S 1997 Attitude determination and control for DARPASAT, a simple spinning spacecraft (AAS 96-077) Adv. Astronaut. Sci. 92 667-81 